\title{
Hunger-promoting AgRP neurons trigger an astrocyte- mediated feed-forward autoactivation loop in mice
}

\author{
Luis Varela,, Bernardo Stutz, ${ }^{1}$ Jae Eun Song, ${ }^{1}$ Jae Geun Kim, ${ }^{2}$ Zhong-Wu Liu, ${ }^{1}$ Xiao-Bing Gao, ${ }^{1}$ and Tamas L. Horvath ${ }^{1}$ \\ 1Program of Integrative Cell Signaling and Neurobiology of Metabolism, Department of Comparative Medicine, Yale School of Medicine, New Haven, Connecticut, USA. ²Division of Life Sciences, \\ College of Life Sciences and Bioengineering, Incheon National University, Incheon, South Korea.
}

\begin{abstract}
Hypothalamic feeding circuits have been identified as having innate synaptic plasticity, mediating adaption to the changing metabolic milieu by controlling responses to feeding and obesity. However, less is known about the regulatory principles underlying the dynamic changes in agouti-related protein (AgRP) perikarya, a region crucial for gating of neural excitation and, hence, feeding. Here we show that AgRP neurons activated by food deprivation, ghrelin administration, or chemogenetics decreased their own inhibitory tone while triggering mitochondrial adaptations in neighboring astrocytes. We found that it was the inhibitory neurotransmitter GABA released by AgRP neurons that evoked this astrocytic response; this in turn resulted in increased glial ensheetment of AgRP perikarya by glial processes and increased excitability of AgRP neurons. We also identified astrocyte-derived prostaglandin $E_{2}$, which directly activated - via EP2 receptors - AgRP neurons. Taken together, these observations unmasked a feed-forward, self-exciting loop in AgRP neuronal control mediated by astrocytes, a mechanism directly relevant for hunger, feeding, and overfeeding.
\end{abstract}

\section{Introduction}

In 2004, we found that the connectivity of hypothalamic feeding circuits is not hardwired but shows predictable changes in response to circulating levels of the metabolic hormones leptin and ghrelin (1). When these dynamic synaptic changes were correlated to behavioral effects of leptin, it became clear that synaptic remodeling evoked by leptin precedes changes in the behavior of animals $(2,3)$. These findings suggested that synaptic plasticity of hypothalamic circuits governed by peripheral metabolic signals is a prerequisite for proper behavioral and autonomic adaptations to the changing peripheral milieu. Further studies, including our own, elaborated on the extent and variety of dynamic synaptic changes in the hypothalamus and beyond in relation to metabolic regulation, including vulnerability to high-fat diet-induced obesity (2, 4-11).

Over the past decade, efforts have been made to identify cellular and molecular determinants of hypothalamic synaptic plasticity, which include putative roles for intracellular signaling molecules such as AMPK (10-12), mitochondrial uncoupling protein 2 (7), and sirtuin 1 (13). Postsynaptic glutamate receptors were also found to be relevant for the synaptic plasticity of agouti-related protein (AgRP) (14) and pro-opiomelanocortin (POMC) neurons (4).

A critical site for control of neuronal output is the perikaryon, where all inputs from dendritic arbors are integrated to impact action potentials that run through axons to affect postsynaptic targets. Thus, the inhibitory input organization of the neuronal perikaryon plays a crucial role in the activation or suppression of axonal action potentials and, in the case of AgRP neurons, in control of

Conflict of interest: The authors have declared that no conflict of interest exists. Copyright: (5) 2021, American Society for Clinical Investigation.

Submitted: September 14, 2020; Accepted: April 8, 2021; Published: April 13, 2021

Reference information: / Clin Invest. 2021;131(10):e144239.

https://doi.org/10.1172/JCl144239. feeding and autonomic output. In our original study, we observed that perikaryal inhibitory input organization of AgRP neurons was readily affected by leptin administration, whereby inhibition of the AgRP system by ghrelin was preceded by increased inhibitory synapses on AgRP neuronal perikarya (1). In subsequent studies, such differences in perikaryal inhibitory inputs of AgRP neurons were also observed between animals that were vulnerable versus resistant to diet-induced obesity (8). In this latter study (8) and further studies $(15,16)$, it was found that the lower number of perikaryal synapses on AgRP neurons was accompanied by increased ensheetment of the cell body membrane by astrocytic processes. These observations suggested that there may be a dynamic relationship between astrocytes and AgRP neurons that control the synaptology and behavioral output of AgRP cells. The current study was undertaken to interrogate this possibility.

\section{Results}

Food restriction induces adaptation of arcuate nucleus astrocytes while impacting inhibitory inputs of AgRP neurons. First, we asked whether food restriction affects arcuate nucleus (ARC) astrocytes. We found that overnight exposure of mice to food deprivation induced a significant increase in the expression levels of glial fibrillary acidic protein (GFAP) in the ARC (Figure 1A). In association with this, we observed more numerous but significantly smaller sized mitochondria in astrocytes of food-deprived animals (Figure 1, B-D, and Supplemental Figure 1, A and B; supplemental material available online with this article; https://doi.org/10.1172/ JCI144239DS1), a sign of enhanced mitochondrial fission (Supplemental Figure 1C). By using electron microscopy, we detected greater glial coverage of AgRP perikarya in food-restricted mice compared with fed controls (Figure 1, E and G). This elevated glial coverage of AgRP neurons was paralleled by changes in their synaptic inputs (Figure 1F and Supplemental Figure 1, D-G). Results 
A

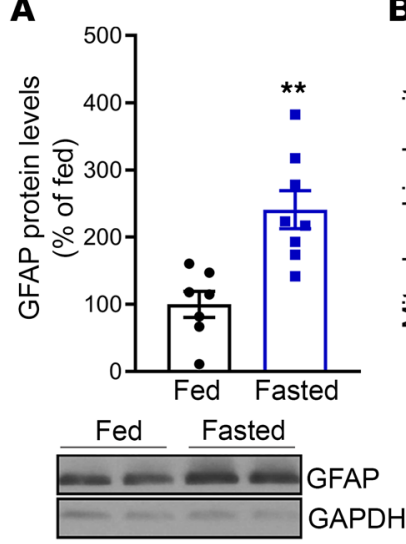

B

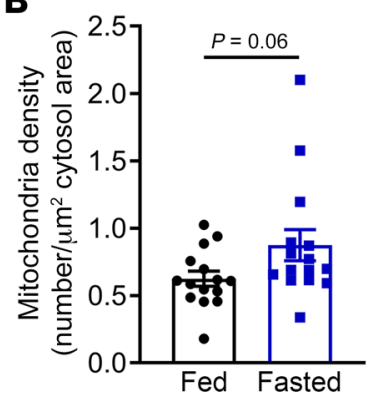

C

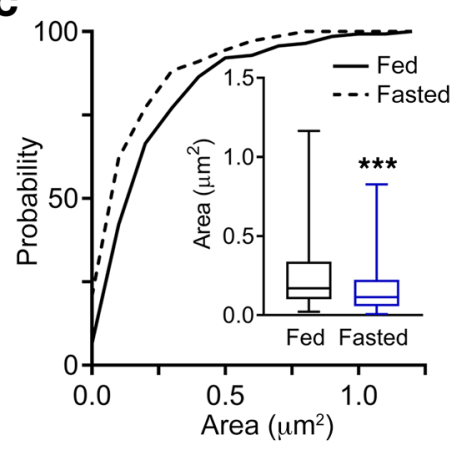

D

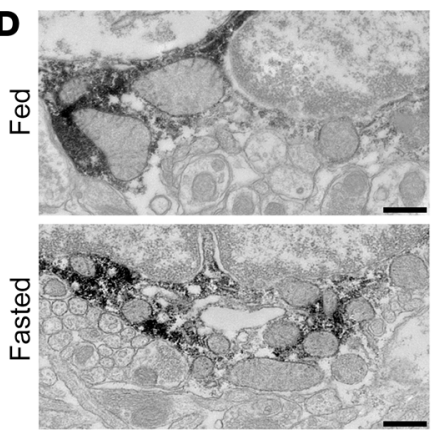

E

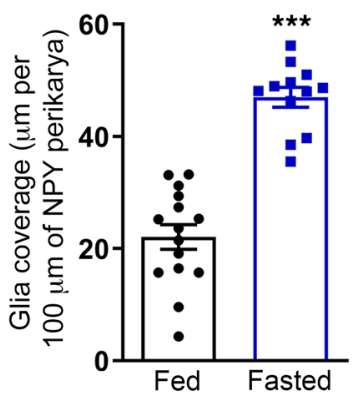

$\mathbf{F}$

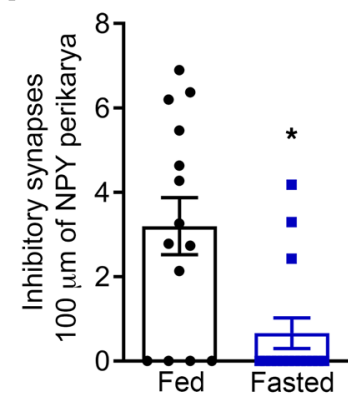

G

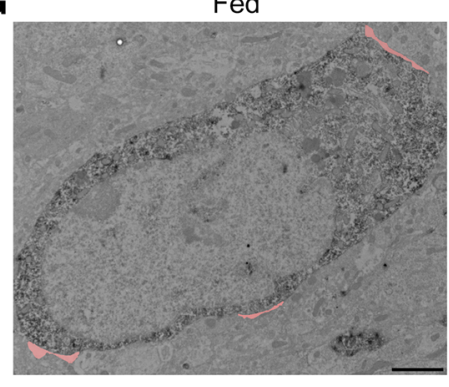

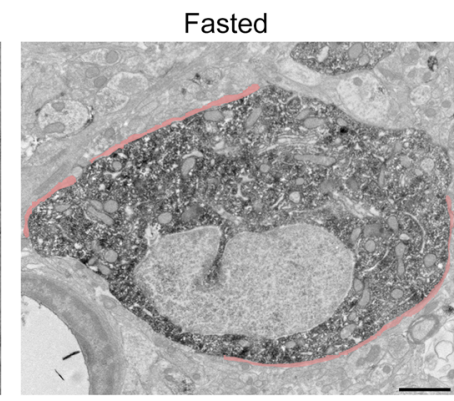

Figure 1. Fasting promotes changes in astrocytes and glial coverage on AgRP neurons. (A) GFAP levels (representative Western blot images and quantification) from MBH of fed and fasted mice ( $n=7 / 8,8-10$ weeks old). (B) Density of mitochondria in Arc astrocytes of fed and fasted mice ( $n=15$ cells per group from 4-5 mice). (C) Cumulative distribution and mean of mitochondrial area in Arc astrocytes of fed and fasted mice ( $n=140 / 145$ mitochondria from 4-5 mice). (D) Representative electron micrographs of mitochondria profiles in Arc astrocytes from fed and fasted mice (scale bars: $500 \mathrm{~nm}$ ). (E) Glial coverage on AgRP cells of fed and fasted mice $(n=15 / 12)$. (F) Inhibitory synapses onto AgRP neurons of fed and fasted mice $(n=14 / 15)$. (G) Representative electron micrographs showing glial coverage onto AgRP neurons from fed and fasted mice. Red traces indicate presence of glial coverage on cell perikarya (scale bars: $2 \mu \mathrm{m}$ ). Data are presented as mean $\pm \mathrm{SEM}$. ${ }^{*} P \leq 0.05,{ }^{* *} P \leq 0.01$, and ${ }^{* *} P \leq 0.001$ as determined by 2 -tailed $t$ test or Kolmogorov-Smirnov test for analyses of cumulative distribution.

corresponding to the electron microscopy observations showed a constellation of miniature events that was consistent with elevated excitability of AgRP neurons in fasted animals (Figure 1F). While the electrophysiological and electron microscopy data corroborate each other, they are not identical. This is not unusual (see our earlier work; ref. 1) in light of the nonoverlapping pitfalls of these techniques. Overall, these results showed that food restriction impacts ARC astrocytes, with concomitant changes in perikaryal synaptic input organization and glial ensheetment of AgRP neurons.

Ghrelin induces adaptation of ARC astrocytes while impacting inhibitory inputs of AgRP neurons. Ghrelin is a gut hormone that is elevated in the circulation during food restriction $(17,18)$, and i.p. administration of ghrelin can rapidly evoke behavioral responses and changes in synaptic organization of AgRP neurons similar to those induced by food restriction (11). Thus, next we explored whether ghrelin elicits astrocytic responses similar to those described above in response to food restriction. Ninety minutes after i.p. ghrelin treatment, we observed increased levels of GFAP (Figure 2A) and more numerous but smaller mitochondria in astrocytes (Figure 2, B-D, and Supplemental Figure 2, A and B). Similarly, we detected elevated glial coverage and a reduced number of symmetrical, putative inhibitory synapses on AgRP perikarya (Figure 2, E-G). Ghrelin treatment decreased the size of neuropeptide Y-expressing (NPY) neuron mitochondria without affecting their number (Figure 2, H-J, and Supplemental Figure 2, C and D). When ghrelin was added to hypothalamic brain slices, it induced a significant depolarization of astrocytic membrane potentials (MPs) in 7 of 9 tested cells (from 7 mice) $(t=3.1001, P<0.05$, paired $t$ test; Figure 3, D and F). Opposite to what takes place in NPY neurons (Figure 3, A-C), blockade of synaptic transmission completely abolished the depolarizing effects of ghrelin on hypothalamic astrocytes in 8 of 8 tested cells (from 6 mice) $(t=0.2261, P=0.83$, paired $t$ test; Figure 3 , E and F). Together, these results showed that ghrelin affects astrocytes indirectly via neuronal mediation.

AgRP neurons mediate ghrelin-induced depolarization of astrocytes while impacting their own inhibitory inputs. AgRP neurons are the likely mediators of ghrelin's action on ARC astrocytes. To test that hypothesis, using a DREADD (designer receptors exclusively activated by designer drugs) approach, we selectively inhibited AgRP neurons during administration of ghrelin (Figure 4A and Supplemental Figure 3). We found that the addition of ghrelin to hypothalamic brain slices of Agrp-Cre mice infected with AAVKORD did not induce a change in the electrical properties of astrocytes in the presence of salvinorin B (SalB) in 8 of 8 tested cells (from 5 mice) $(t=0.9520, P=0.37$, paired $t$ test; Figure $4 \mathrm{~B})$. 

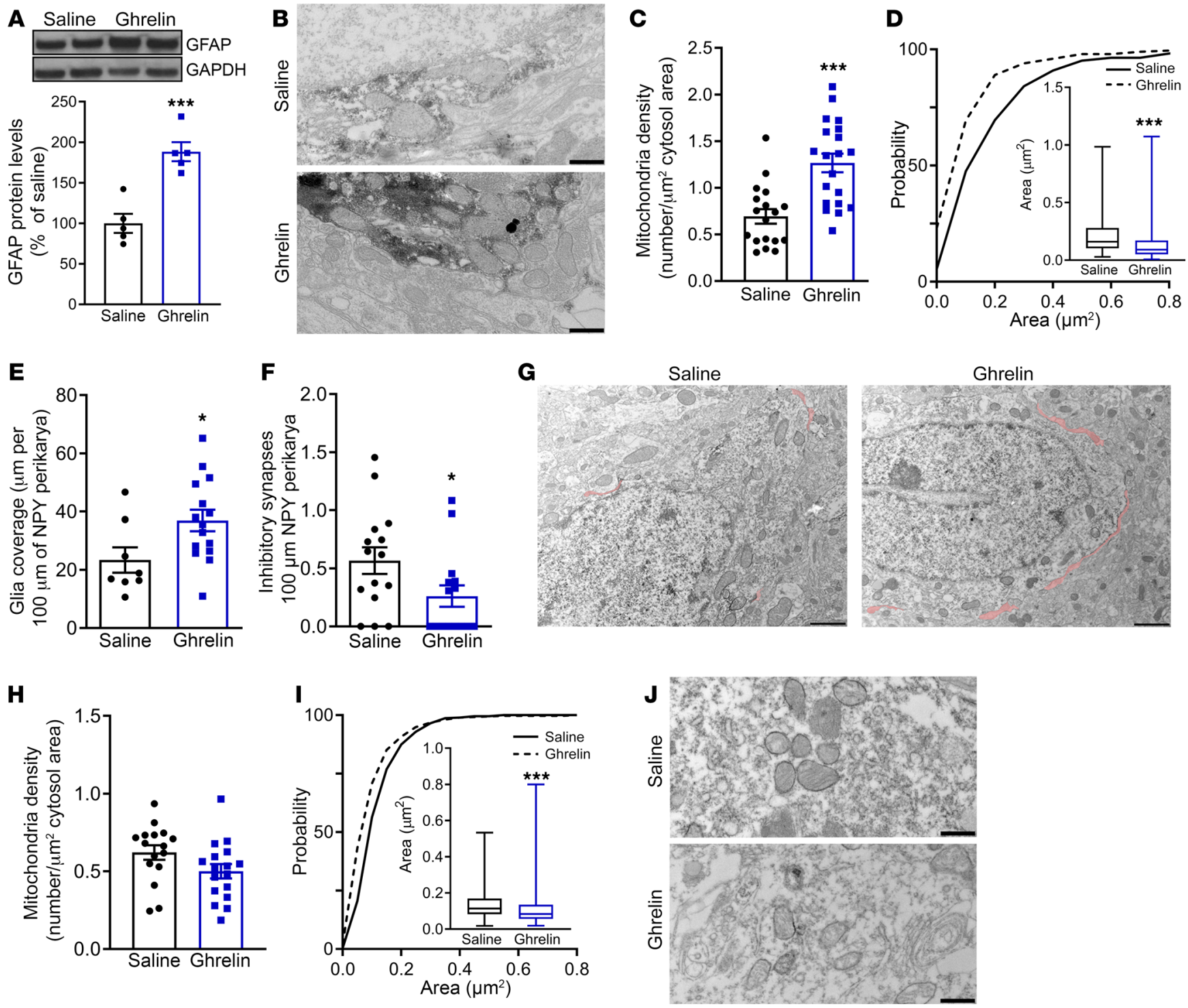

Figure 2. Ghrelin promotes changes in astrocytes and glial coverage on AgRP neurons. (A) GFAP levels (representative Western blot images and quantification) from MBH of saline- and ghrelin-treated mice $(n=5 / 5)$. (B) Representative electron micrographs of mitochondria profiles in Arc astrocytes from saline- and ghrelin-treated mice (scale bars: $500 \mathrm{~nm}$ ). (C) Mitochondrial density in Arc astrocytes of saline- and ghrelin-treated mice $(n=18 / 20$ cells from 4 mice per group). (D) Cumulative distribution and mean of mitochondrial area in Arc astrocytes of saline- and ghrelin-treated mice ( $n=164 / 199$ mitochondria). (E) Glial coverage on AgRP cells of saline- and ghrelin-treated mice $(n=8 / 15)$. (F) Inhibitory synapses onto AgRP neurons of saline- and ghrelintreated mice $(n=15 / 15)$. (C) Representative electron micrographs showing glial coverage on AgRP neurons from saline- and ghrelin-treated mice. Red traces indicate presence of glial coverage on cell perikarya (scale bars: $2 \mu \mathrm{m})$. (H) Mitochondrial density in NPY cells of saline- and ghrelin-treated mice ( $n$ $=16 / 17$ cells). (I) Cumulative distribution and mean of mitochondrial area in NPY neurons of saline- and ghrelin-treated mice $(n=463 / 370$ mitochondria). (J) Representative electron micrographs of mitochondria profiles in NPY neurons from saline- and ghrelin-treated mice (scale bars: $500 \mathrm{~nm}$ ). Data are presented as mean \pm SEM. ${ }^{*} P \leq 0.05$ and ${ }^{* * *} P \leq 0.001$ as determined by 2 -tailed $t$ test or Kolmogorov-Smirnov test for analyses of cumulative distribution.

To further analyze the role of AgRP neurons in the adaptation of local astrocytes, we applied DREADD technology to selectively activate AgRP neurons. After selective activation, we observed an increase in GFAP content in the mediobasal hypothalamus (Figure 4C) and more numerous but smaller mitochondria in local astrocytes (Figure 4, E-G, and Supplemental Figure 4, B and C). We also found increased glial coverage and a concomitant decrease in symmetrical, putative inhibitory synapses on AgRP perikarya upon their own chemogenetic activation (Figure 4, $\mathrm{H}-\mathrm{J}$ ). Moreover, selective activation of AgRP neurons resulted in smaller mitochon- dria in these neurons (Figure 4, K-M, and Supplemental Figure 4, D and E). These alterations in mitochondrial morphology in both cell types after selective AgRP activation are a sign of enhanced fission, as illustrated by increased protein levels of pDRP1 in the mediobasal hypothalamus (MBH) of these mice (Supplemental Figure $4 \mathrm{~F}$, $n=7 / 8$ mice). Addition of CNO to the hypothalamic brain slices of $h M 3 D_{G q}{ }^{A g R P}$ mice produced significant depolarization of astrocytic MP in 6 of 6 cells (from 4 mice) $(t=2.022, P<0.05,1$-tailed paired $t$ test; Figure $4 \mathrm{D})$. Together, these results show that AgRP neurons mediate the effect of ghrelin on ARC astrocytes. 
A

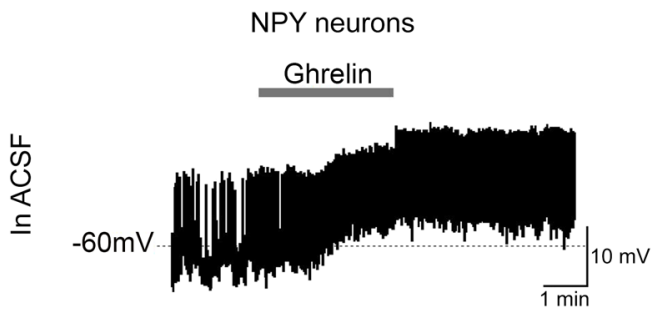

B
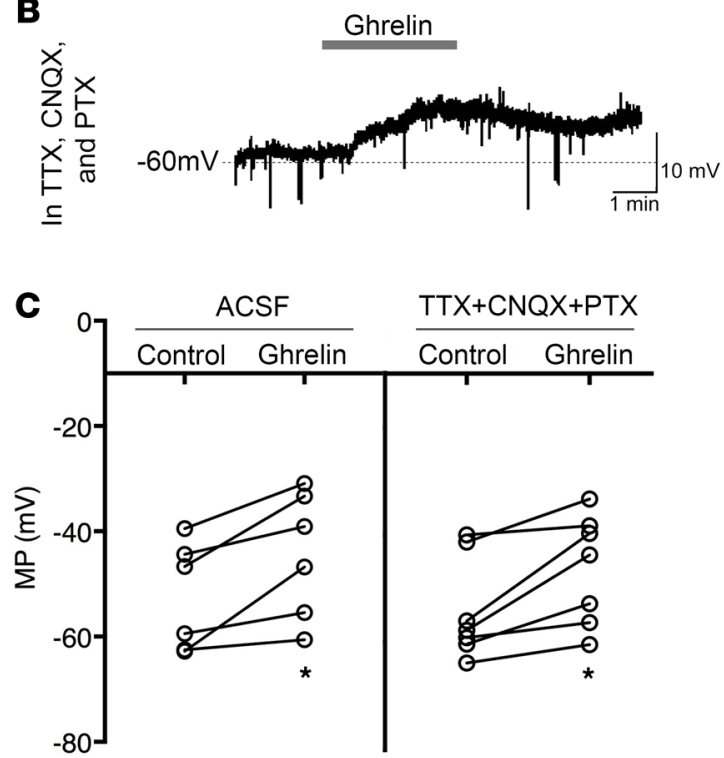

D

Astrocytes

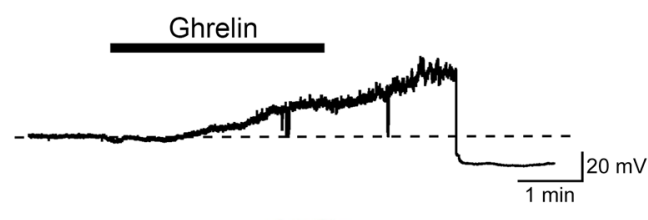

$\mathbf{E}$

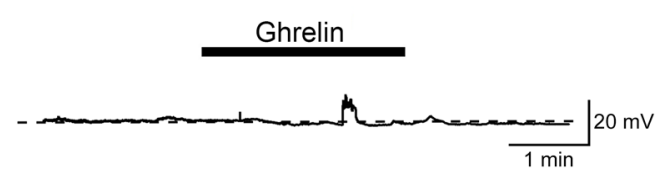

$\mathbf{F}$

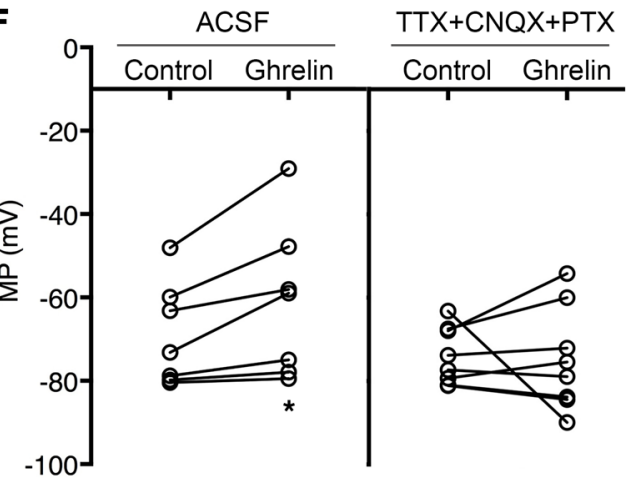

Figure 3. Ghrelin causes depolarization in astrocytes, and this depends on neuronal activity. Representative traces of AgRP neurons in response to ghrelin in (A) ACSF ( $n=6$ of 6 cells from 4 mice) and (B) tetrodotoxin (TTX) plus PTX plus cyanquixaline (CNQX) ( $n=7$ of 7 cells from 4 mice). (C) MP of AgRP neurons exposed to ghrelin in the presence of ACSF or TTX+PTX+CNQX. Representative traces of hypothalamic astrocytes in response to ghrelin in ACSF (D) ( $n=7$ of 9 cells from 7 mice) and) TTX + PTX + CNQX (E) $(n=8$ of 8 cells from 6 mice). (F) MP of hypothalamic astrocytes exposed to ghrelin in the presence of ACSF or TTX+PTX+CNQX. Data are presented as mean \pm SEM. ${ }^{*} P \leq 0.05$ as determined by 2 -tailed $t$ test.

AgRP-released GABA mediates the depolarizing effect of ghrelin on astrocytes. Changes in AgRP neuron activity alter the release of different neuropeptides, including AgRP, NPY, and GABA (19). Our next goal was to identify the factor mediating the communication between AgRP neurons and astrocytes. We carried out electrophysiological studies and observed that whereas addition of AgRP (in 5 of 5 cells from 3 mice) or NPY (in 5 of 5 cells from 3 mice) to hypothalamic brain slices did not produce any changes in the electrical properties of astrocytes (Figure 5, A and B), GABA caused strong and rapid depolarization of the astrocytic MP in 5 of 5 cells from 4 mice $(t=4.4191, P<0.05$, paired $t$ test; Figure 5C). Moreover, we analyzed RNA specifically from hypothalamic astrocytes and found that fasting exposure increased expression levels of Gabarap in these cells (Figure 5D). Next, we investigated whether selective blockade of GABA receptors impacts the above findings on astrocytes. In the presence of picrotoxin (PTX; a GABAaR antagonist), the addition of ghrelin to hypothalamic brain slices did not trigger changes in the electrical properties of astrocytes in 6 of 6 tested cells (from 4 mice; Figure 5E). Likewise, $\mathrm{CNO}$ failed to depolarize hypothalamic astrocytes from $h M 3 D_{G q}{ }^{A g R P}$ mice pretreated with PTX in 8 of 8 cells (from 6 mice; Figure $5 \mathrm{~F}$ ). These findings pointed to AgRP-released GABA as the effector of ghrelin-induced astrocyte depolarization.
Astrocyte-released prostaglandin $E_{2}$ activates AgRP neurons through its effects on EP2. Various studies have shown that prostaglandin $\mathrm{E}_{2}$ (PGE2) mediates communication between astrocytes and diverse subsets of hypothalamic neurons (20, 21). Astrocyte-released PGE2 directly binds the EP receptors expressed in neurons to modulate their activity (20). Thus, we investigated whether PGE2 may be relevant for modulation of AgRP neurons by ghrelin. First, we found that acute central administration of PGE2 $(100$ pmol) resulted in increased AgRP activation as assessed by c-Fos expression (Figure 6, A and B). PGE2-activated AgRP cells contained smaller and rounder mitochondria (Figure 6, C-G), consistent with the results above regarding ghrelin. Electrophysiological recordings demonstrated a direct excitatory effect of PGE2 on AgRP neurons in 9 of 9 tested cells (from 4 mice), which was mediated by the EP2 receptor (Supplemental Figure 5, A-C). In addition, previously reported astrocyte-mediated NPY neuron activation (22) was abolished in the presence of the EP2 receptor inhibitor PF04418948 (PF). In NPY-GFP mice expressing AAVGFAP-hM3D $_{\mathrm{Gq}}$-mCherry, application of CNO $(10 \mu \mathrm{M})$ through bath solution induced a significant depolarization (baseline -56.7 $\pm 1.7 \mathrm{mV}, \mathrm{CNO}-47.6 \pm 2.9 \mathrm{mV}$, washout $-55.7 \pm 1.2 \mathrm{mV}, n=5$ from 4 mice; $F_{2,8}=7.494, P=0.0147$, 1-way repeated-measures ANOVA; post hoc Tukey's test, control vs. CNO, $q=5.000, P=0.0187$; 
$\begin{array}{cccc}\mathrm{A} & \mathrm{Ctl}+ & \mathrm{Ctl}+ & h M 4 D_{G i}^{\mathrm{AgRP}+} \\ \text { Saline } & \text { Ghrelin } & \text { Ghrelin }\end{array}$

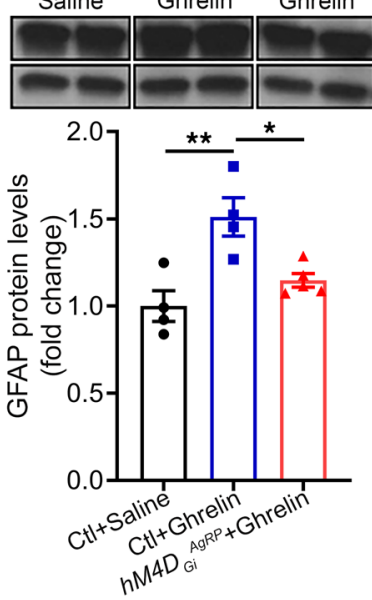

E

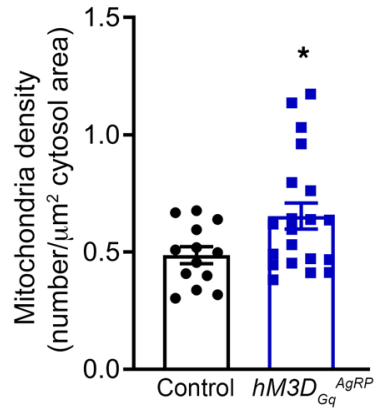

F

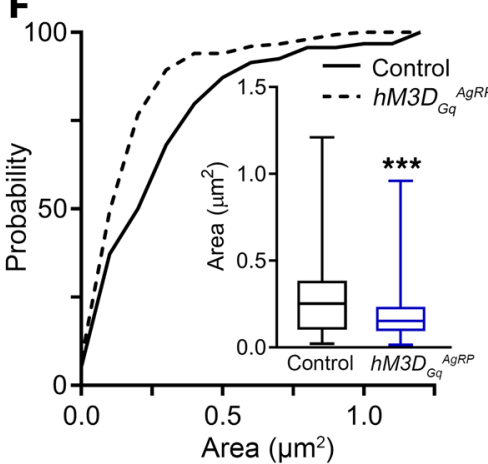

$\mathbf{K}$

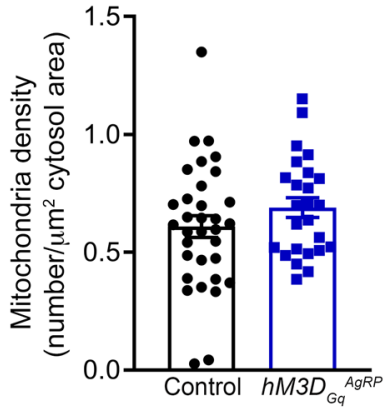

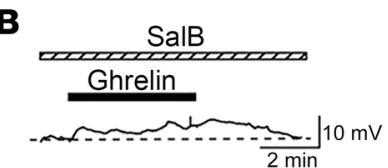

GAPDH

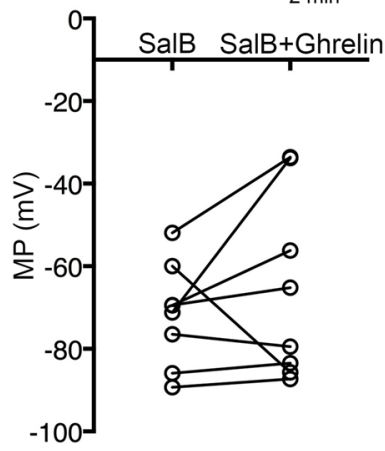

C
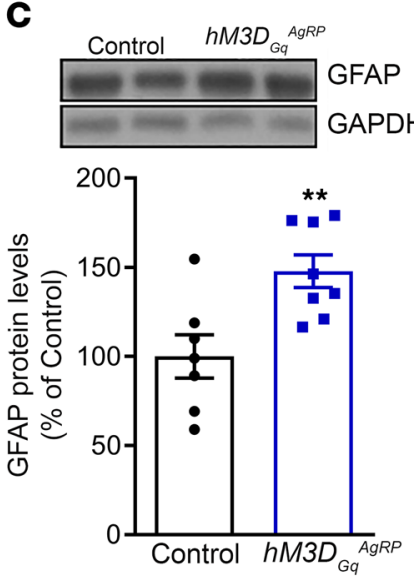
GFAP
D GAPDH

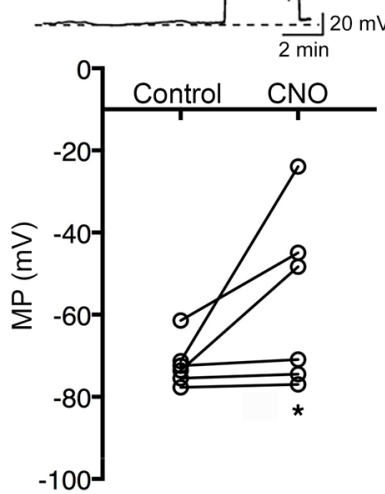

G
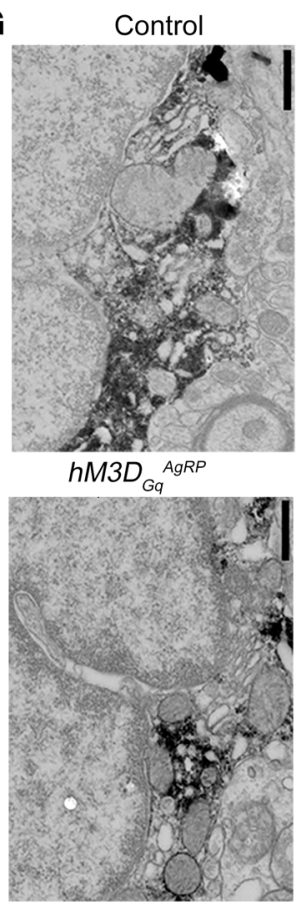

L

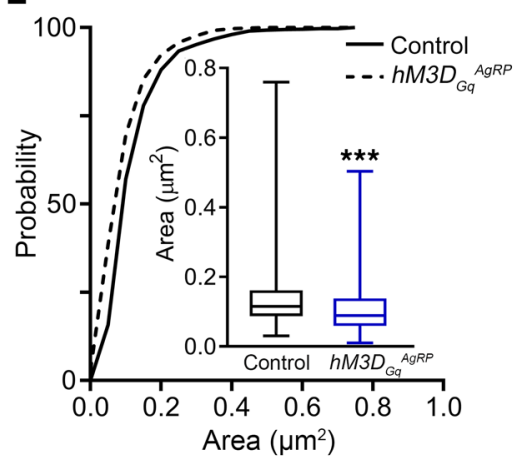

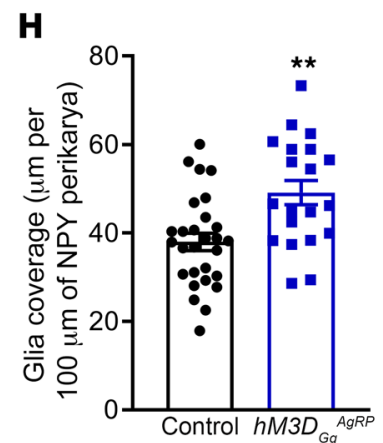

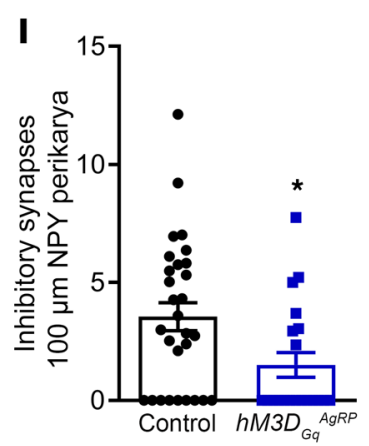

$\mathbf{M}$
$\mathbf{J}$
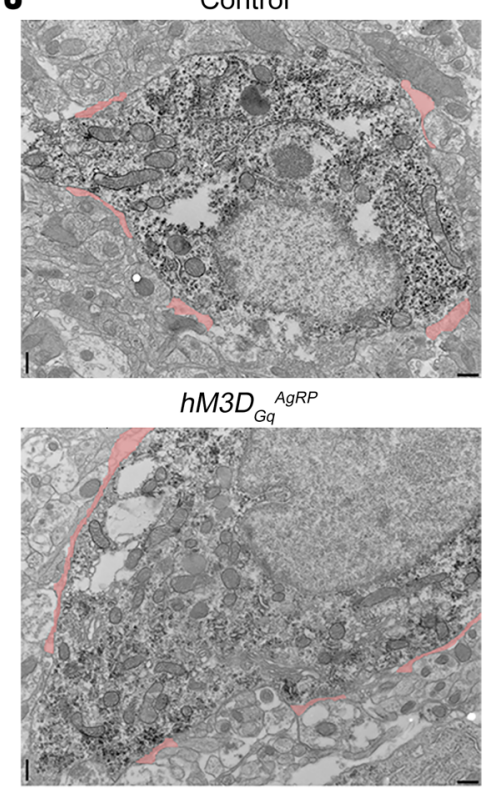

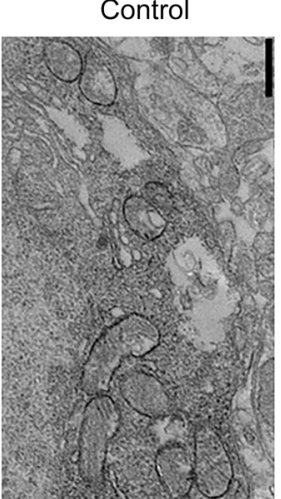

$h M 3 D_{G q}^{A g R P}$

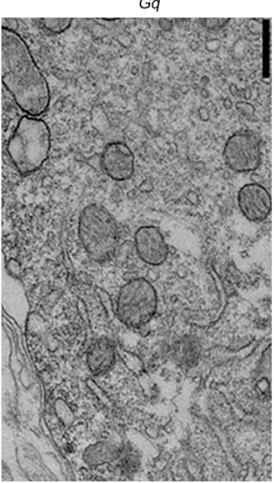


Figure 4. AgRP activation promotes changes in astrocytes and glial coverage on AgRP neurons. (A) GFAP levels (representative Western blot and quantification) from MBH of control (Ctl) and $h M 4 D_{G i}{ }^{A g R P}$ mice treated with either saline or ghrelin $(n=4 / 4 / 5)$ (lanes were run on the same gel but were noncontiguous). (B) Representative trace and MP of hypothalamic astrocytes from AAV-HA-KORD-IRES-mCitrine-infected AgRP-Cre mice exposed to ghrelin in the presence of SalB ( $n=8,8$ tested cells, 5 mice). (C) GFAP levels (representative Western blot images and quantification) of $\mathrm{MBH}$ from control and $h M 3 D_{G q}{ }^{A g R P}$ mice $(n=7 / 7)$. (D) Representative trace and MP of hypothalamic astrocytes from $h M 3 D_{G q}{ }^{A g R P}$ mice exposed to CNO ( $n=6,6$ cells from 4 mice). (E) Mitochondrial density and (F) cumulative distribution and mean of mitochondrial area in Arc astrocytes of control and $h M 3 D_{C q}{ }^{A g R P}$ mice ( $n=95 / 147$ mitochondria, $13 / 20$ cells, 3-4 mice). (G) Representative electron micrographs of mitochondria profiles in Arc astrocytes from control and $h M 3 D_{G q}{ }^{A g R P}$ mice (scale bars: $500 \mathrm{~nm}$ ). (H) Glial coverage and (I) inhibitory synapses onto AgRP/NPY neurons of control and $h M 3 D_{G q}{ }^{A g R P}$ mice ( $n=28 / 20$ cells, 5-6 mice). (J) Representative electron micrographs showing glial coverage on AgRP/NPY neurons from control and $h M 3 D_{C q}{ }^{A g R P}$ mice. Red traces indicate presence of glial coverage on cell perikarya (scale bars: $2 \mu \mathrm{m}$ ). (K) Mitochondrial density and (L) cumulative distribution and mean of mitochondrial area in AgRP/NPY neurons of control and $h M 3 D_{G q}{ }^{A g R P}$ mice ( $n=709 / 665$ mitochondria, 33/25 cells, 5-6 mice). (M) Representative electron micrographs of mitochondrial profiles in AgRP/NPY neurons from control and $h M 3 D_{G q}{ }^{A g R P}$ mice (scale bars: $500 \mathrm{~nm}$ ). Data are presented as mean $\pm \mathrm{SEM} .{ }^{*} P \leq 0.05,{ }^{* *} P \leq 0.01$, and ${ }^{* * *} P \leq 0.001$ as determined by 2-tailed $t$ test, 1-way ANOVA, or Kolmogorov-Smirnov for analyses of cumulative distribution.

control vs. washout, $q=0.5691, P=0.9155 ; \mathrm{CNO}$ vs. washout, $q=$ 4.431, $P=0.0334$ ) in NPY-GFP neurons in the ARC (Figure 6H). In the presence of EP2 receptor inhibitor, application of $\mathrm{CNO}$ induced mild changes in $\mathrm{MP}$ ( $\mathrm{PF}$ alone, $-50.9 \pm 1.1 \mathrm{mV} ; \mathrm{PF}+\mathrm{CNO}$, $-49.0 \pm 0.6 \mathrm{mV}$; PF washout, $-51.5 \pm 2.2 \mathrm{mV} ; n=6$ from 3 mice), which was not statistically significant $\left(F_{2,10}=1.564, P=0.2564\right.$, 1-way repeated-measures ANOVA; Figure 6I). Finally, central administration of PF blocked ghrelin-induced feeding (Figure 6J).

\section{Discussion}

Over the past decades, our understanding of glial cell function in the brain has evolved significantly. The role of glial cells in various aspects of control of neuronal circuits has been recognized (23-27). Astrocytes specifically have been tied to both pre- and postsynaptic control of neuronal transmission via multimodal action $(23,25,28,29)$. We and others have shown, in the hypothalamus, the involvement of both astrocytes and microglia in synaptic input organization and activity of neuronal circuits affecting feeding, energy, and glucose metabolism $(8,15,16,30)$. The results described herein show that AgRP neurons can directly stimulate neighboring astrocytes, revealing neuron-to-astrocyte communication in the physiological adaptations to changing energetic status. Previous reports have described that in hippocampal and cortical areas, neurons communicate with adjacent astrocytes by releasing various neurotransmitters (29, 31-33). Among them, GABA has been shown to be a main neuronderived factor that triggers the depolarization of astrocytes (3436). Indeed, we demonstrated that AgRP neuron-released GABA is the main mediator of this cell-to-cell communication in the hypothalamus. The presence of PTX in the bath completely suppressed the action of ghrelin and the selective activation of AgRP neurons on hypothalamic astrocytes.
Our study also revealed that AgRP-activated astrocytes could signal to AgRP neurons in a dual manner. First, astrocytic processes replaced inhibitory connections on AgRP perikarya, as assessed by electron microscopy, and changed the excitability of these neurons, as shown by miniature inhibitory postsynaptic currents. A decade ago, we showed the involvement of hypothalamic astrocytes in the morphological synaptic plasticity of AgRP and POMC neurons (8). In the present study, we found that fasting, ghrelin administration, or selective activation of AgRP neurons caused activation of glial cells by GABA and an increase in glial coverage of AgRP perikarya, with a concomitant reduction in the number of synapses on their plasma membrane. Besides this morphological plasticity of AgRP neurons, we also explored whether glial cell-derived signals have a direct effect on AgRP neuronal activity. Prostaglandins are released by hypothalamic astrocytes and can stimulate the activity of various neuronal populations $(20,21)$. Beyond their role in sickness behavior, data indicate that local actions of prostaglandins in the CNS are necessary for maintaining basic physiological functions $(20,37)$. In line with this, we found that PGE2 promotes the activation of AgRP cells through their actions specifically on EP2 receptors. Blockade of EP2 abolished astrocyte activation of AgRP neurons and feeding response to ghrelin. Thus, these data provide a mechanism by which hypothalamic astrocytes may be affecting AgRP cells. We do not suggest that PGE2 is involved in the glial changes that affect AgRP neuron input organization. However, our data suggest that at least in the initial phase of ghrelin- or fasting-triggered AgRP neuronal activation, when glial processes move to cover AgRP perikarya, PGE2 production in astrocytes and their potential to act on AgRP neurons are temporarily and topographically aligned (Figure 7).

AgRP neurons are considered "first-order" sensory neurons in the regulation of feeding, as they mediate information on the systemic energetic state to a broad array of brain circuits that, in turn, alters complex behaviors $(11,38-41)$. In this regard, that these neurons are characterized by rapid synaptic plasticity in response to the changing metabolic environment is reasonable and essential to assuring that peripheral metabolic information can be conveyed to evoke feeding in support of survival, with limited interference by inhibitory signals arising from other brain areas. While a feedback mechanism was proposed to explain the increase in excitatory input of AgRP neurons to promote feeding (11), how synaptic plasticity of inhibitory inputs on AgRP perikarya is controlled has been unresolved. Gating of neuronal perikarya by inhibitory inputs is crucial for filtering excitation-evoked firing of effector neurons, such as the principal neurons of the cortex and hippocampus $(5,42-45)$. This assures appropriate control of "noise" in support of predictable orchestration of behavioral and autonomic output. The necessity of such gating may be immediately obvious, for example, in the case of motor neurons or pyramidal cells of the cortex, which compute vast amounts of information arising from different brain sites. On the other hand, there are certain neuronal populations, such as lateral hypothalamic hypocretin/orexin neurons, that are dominated by excitatory inputs even at the perikarya (46). This makes sense, as noise by definition serves as a signal for these neurons, which are critical for arousal (46). In the case of AgRP neurons, the most crucial information flow arises from the periphery to trigger their activation in support of feeding, which 
A

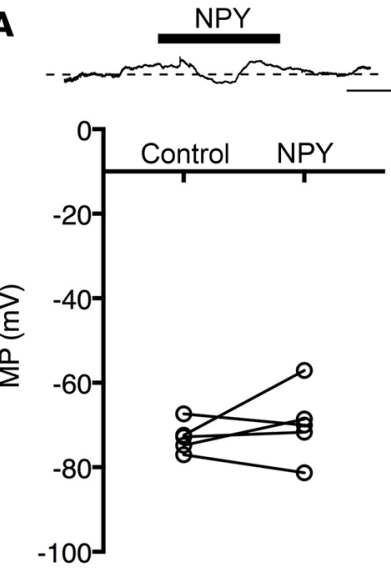

D

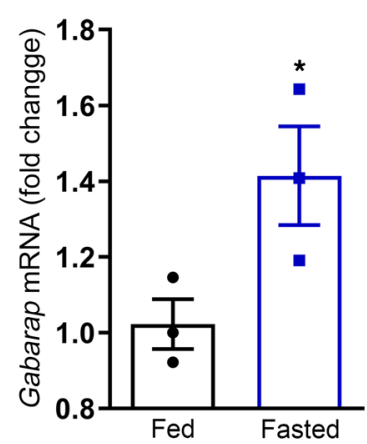

B
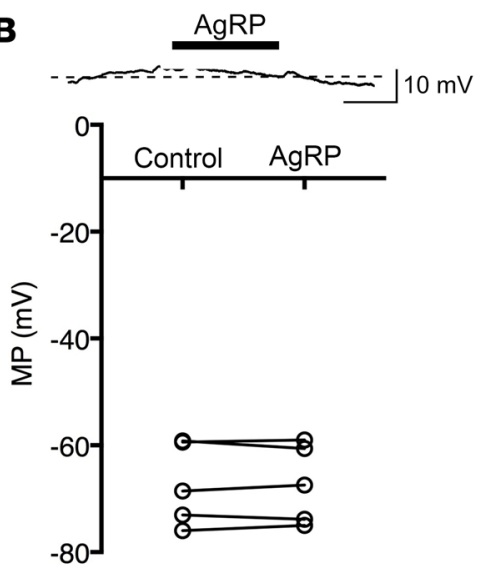

E

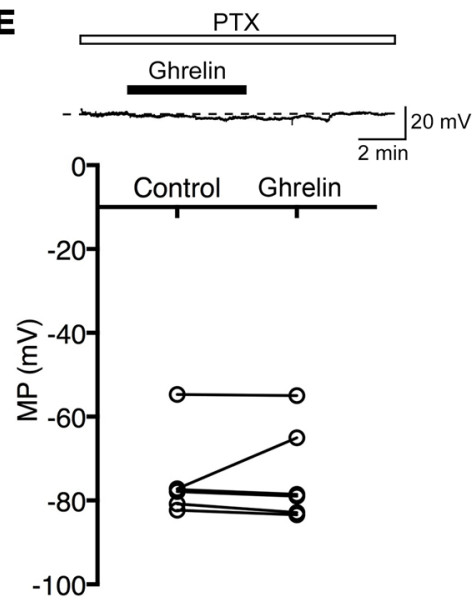

C

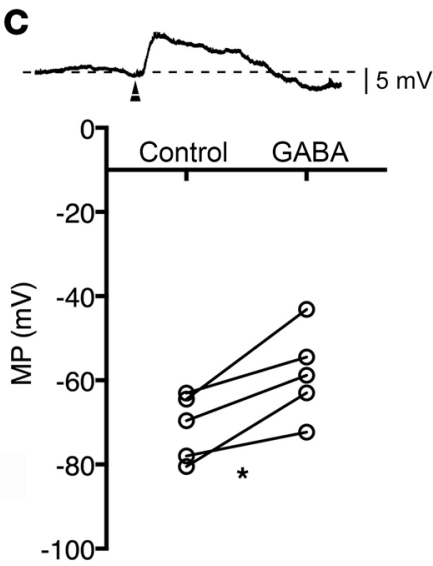

$\mathbf{F}$

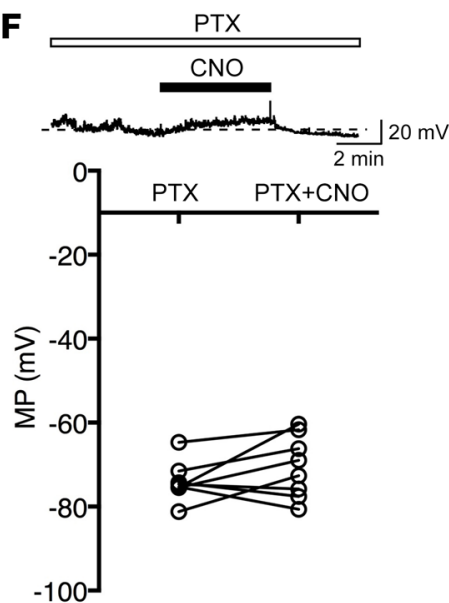

Figure 5. GABA, but not NPY or AgRP, depolarizes Arc astrocytes. (A-C) Representative traces and MPs of hypothalamic astrocytes exposed to NPY ( $n=5$ of 5 cells, 3 mice), AgRP ( $n=5$ of 5 cells from 3 mice), and GABA ( $n=5$ of 5 cells, 4 mice). (D) Astrocyte-specific Gabarap mRNA levels from fed and fasted mice $(n=3 / 3$ ). (E) Representative trace and MP of hypothalamic astrocytes exposed to ghrelin in the presence of CABA receptor blocker ( $n=6$ of 6 cells, 4 mice). (F) Representative trace and MP of hypothalamic astrocytes from $h M 3 D_{G q}{ }^{A g R P}$ mice exposed to CNO in the presence of GABA receptor blocker ( $n=8$ of 8 cells, 6 mice). Data are presented as mean \pm SEM. ${ }^{*} P \leq 0.05$ as determined by 2 -tailed $t$ test.

is crucial for survival $(1,2,7)$. A dominant component of input on peripheral metabolic state of these neurons is circulating hormone and nutrient signals. Thus, morphological elimination of gating inhibitory synapses by the process described herein fits well with the biological necessity for AgRP neuronal activation to support survival, but it may also be relevant for pathological processes associated with obesity.

\section{Methods}

Animals and surgery. Mice were kept under standard laboratory conditions with free access to standard chow food and water, unless otherwise stated. Seven- to 9-week-old male mice were randomized and used for feeding experiments and all experimental studies. Npy$g f p$ (no. 008321), Gfap-gfp (no. 003257), Agrp-Cre (no. 012899), and $\mathrm{R} 26-\mathrm{hM} 4 \mathrm{D}_{\mathrm{Gi}} / \mathrm{mCitrine}$ (no. 026219) transgenic mice were purchased from the Jackson Laboratory. $h M 3 D_{G q}{ }^{A g R P}$ mice were provided by J.C. Bruning and S.M. Steculorum (Max Planck Institute for Metabolism Research, Cologne, Germany) (47). Ghsr-KO mice and their littermates were obtained as previously described (7). Gfap-gfp, Agrp-cre, $h M 4 D_{G i}^{A g R P}$, and $h M 3 D_{G q}^{A g R P}$ mice were maintained on a mixed background in our laboratory. Mice of the Ghsr-KO line were maintained on a C57BL/6 background. Gfap-gfp mice were crossed with the $h M 3 D_{G q}{ }^{A g R P}\left(G f a p-g f p h M 3 D_{G q}^{A g R P}\right)$ mouse strain to allow visualization of astrocytes for electrophysiological recordings. $N p y$ - $g f p$ mice were crossed with the $h M 3 D_{G q}{ }^{A g R P}$ ( $N p y$-gfp $h M 3 D_{G q}{ }^{A g R P}$ ) mouse strain to enhance the signal for electron microscopy purposes.

Cannula placement. Cannulas (PlasticOne) were implanted i.c.v. at least 4 days before the experiment. Buprenorphine was administered 30 minutes before surgery, and mice were anesthetized with ketamine. Mice were placed in a stereotaxic instrument that used a platform specifically designed by the manufacturer (Kopf Instruments) for mouse surgery. Craniotomy was achieved through use of a sterile 25-gauge needle; a 33-gauge (sterile) stainless steel, single-guide cannula was placed unilaterally at $1.2 \mathrm{~mm}$ lateral and $1 \mathrm{~mm}$ posterior from the bregma. Cranioplastic cement and mounting screws were used to fix the cannulas. The incision was closed with a sterile surgical clip. Body temperature and respiratory rate were monitored throughout both procedures. Food and water intake as well as general and cannula appearance were checked 3 days after surgery as part of postoperative care.

Stereotaxic virus injection. Animals were administered injections as described previously $(22,48)$. In brief, bilateral virus injections were 
A

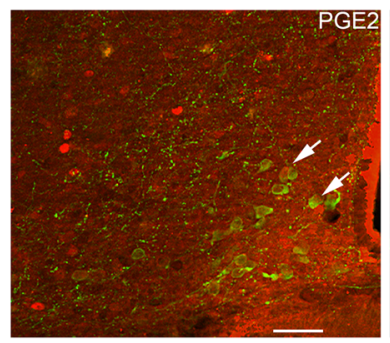

C

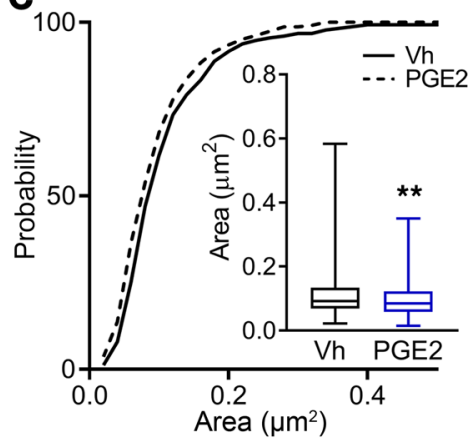

E

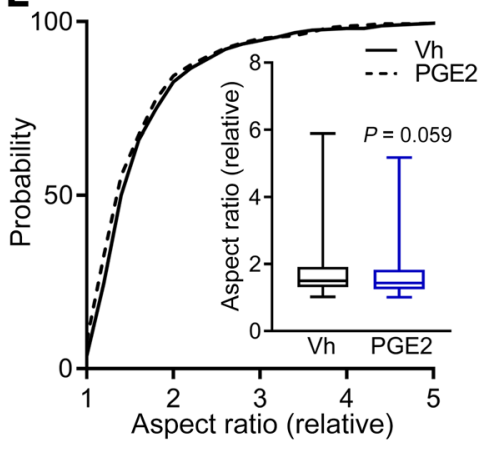

H

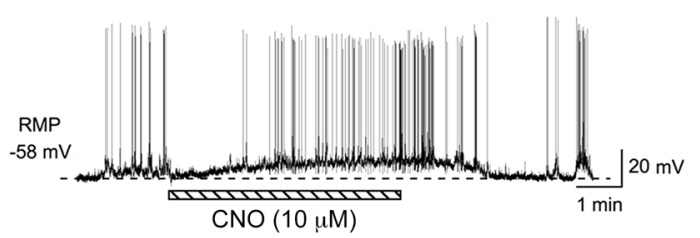

I

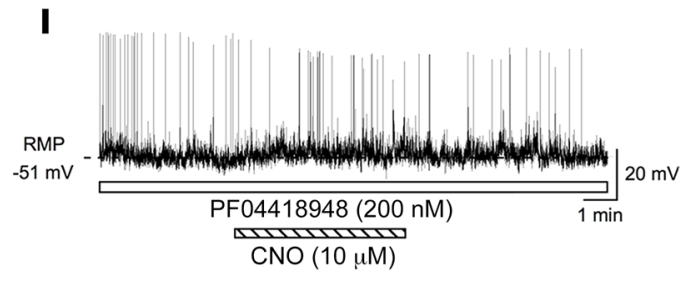

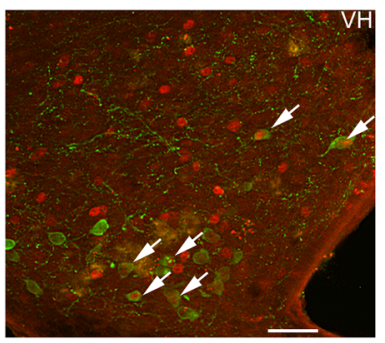

D

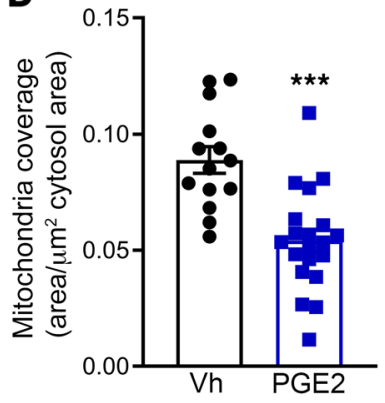

$\mathbf{F}$

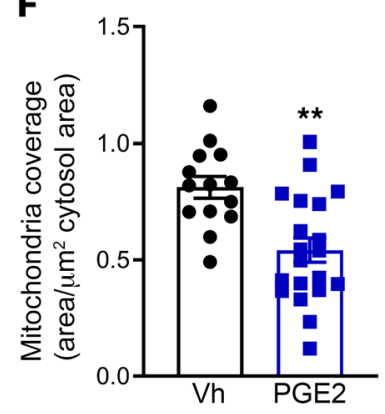

Vh

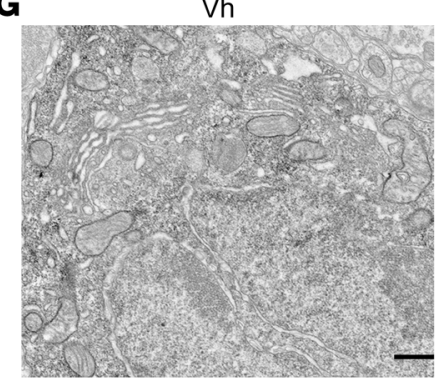

PGE2

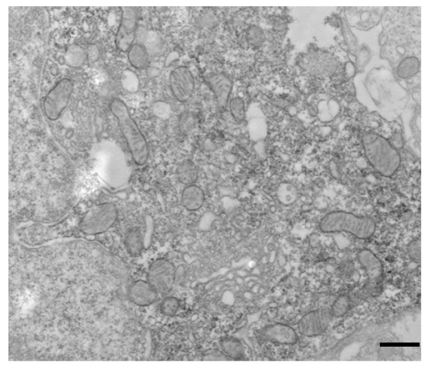

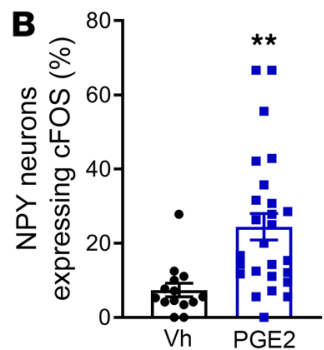

G
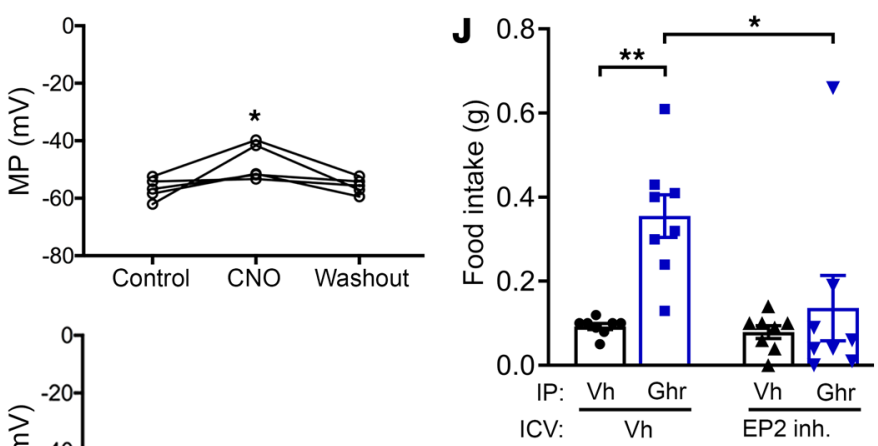

Figure 6. PGE2 activates NPY neurons through its action on EP2. (A) Representative images and (B) graph showing the colocalization of NPY neurons and c-Fos (scale bar: $50 \mu \mathrm{m}$ ) of PGE2- and vehicle-treated (VH-treated) mice ( $n=14 / 26$ slices, 3-4 mice). Arrows denote colocalization between c-Fos (red) and NPY neurons (green). Mitochondrial area (C), coverage (D), aspect ratio (E), and density (F) in AgRP/NPY cells of PGE2- and vehicle-treated mice $(n=$ 402/473 mitochondria, 14/26 cells from 3-4 mice). (G) Representative electron micrographs of mitochondria in AgRP/NPY neurons from mice treated with i.c.V. PGE2 and vehicle (scale bars: $500 \mathrm{~nm}$ ). (H) Left: Representative trace of an NPY-GFP neuron before and during activation of Arc astrocytes by CNO and after washout. Right: MP of Arc NPY-CFP neurons $(n=5)$ before (Control) and during activation of Arc astrocytes by CNO and after washout. (I) Left: Representative trace of an NPY-GFP neuron before and during activation of Arc astrocytes by CNO and after washout while exposed to the EP2 receptor inhibitor PF0441894 (PF). Right: MP of Arc NPY-GFP neurons $(n=6)$ before PF and during activation of Arc astrocytes by CNO and after washout while exposed to the EP2 receptor inhibitor PF. (J) Food intake (1 hour) after the administration of i.p. ghrelin or vehicle. Fifteen minutes prior to the i.p. delivery of ghrelin (Ghr) or vehicle, mice were treated with i.c.v. PF or vehicle ( $n=8$ mice per group). Data are presented as mean $\pm S E M .{ }^{*} P \leq 0.05$, ${ }^{* *} P \leq 0.01$, and ${ }^{* * *} P \leq$ 0.001 as determined by 2 -tailed $t$ test or Kolmogorov-Smirnov test for analyses of cumulative distribution. For analyses of 2 groups and 2 conditions, 2-way ANOVA with post hoc Tukey's test was performed (J). One-way ANOVA for experiments with 3 independent groups (H and I). 


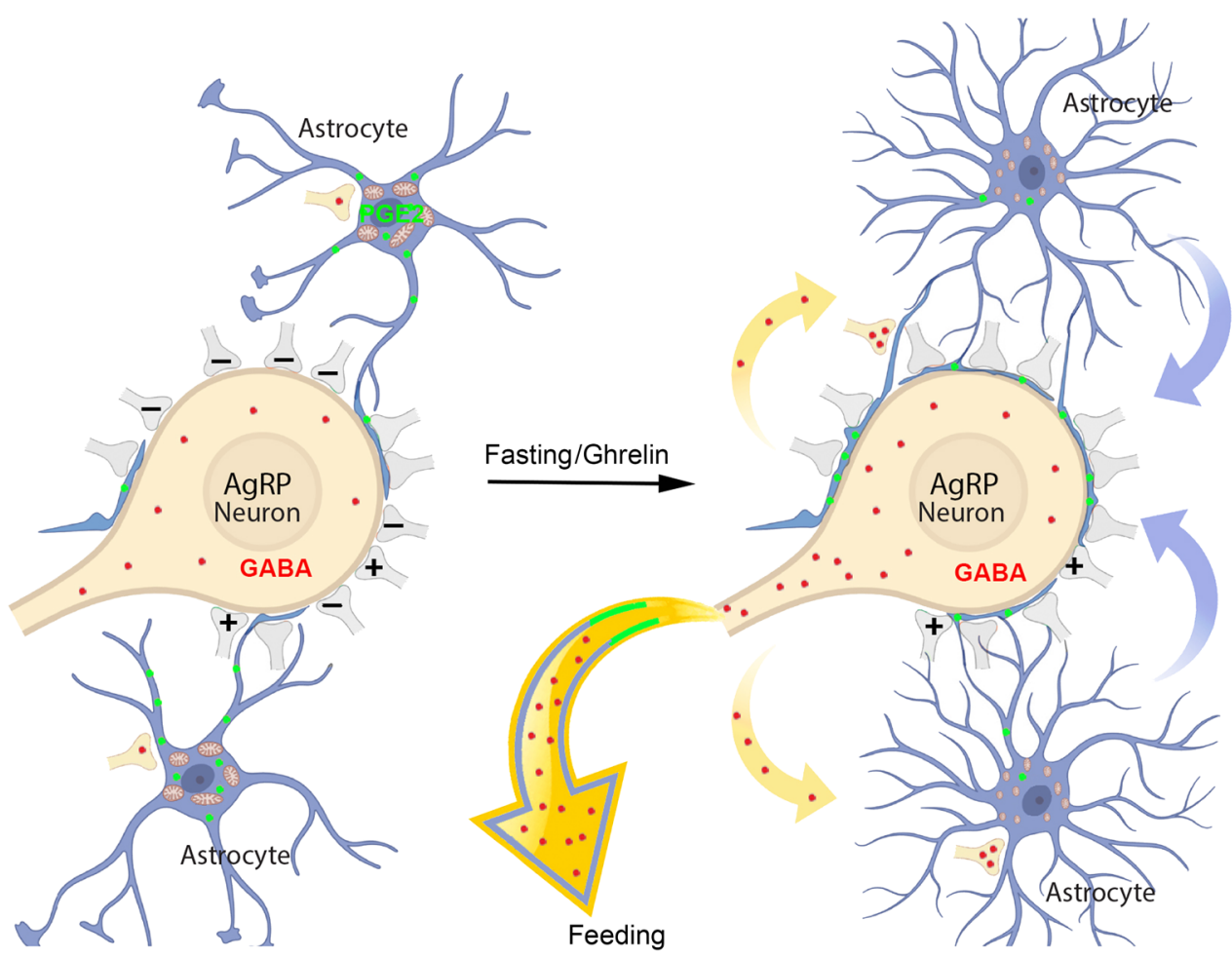

Figure 7. Illustration of key findings of the study. Our observations showed that activation (by fasting, ghrelin administration, or chemogenetics) of AgRP neurons (which are GABAergic neurons) activates neighboring glial cells via GABAergic transmission. Activated glial cells have smaller mitochondria, and their processes increase the ensheetment of AgRP neuronal perikarya, resulting in synaptic input organization of these cells that is consistent with their increased excitability and promotion of feeding. Our data also show that PCE2 signaling of glial origin may have a role in the activation of AgRP neurons in this process. Image created with Biorender.com. made into the ARC of anesthetized 6-week-old male Gfap-gfp Agrpcre and Npy-gfp mice, placed in a stereotaxic apparatus (model 902; Kopf Instruments). Viruses $(500 \mathrm{~nL})$ containing the inhibitory DREADDs rAAV9/AAV-HA-KORD-IRES-mCitrine (ref. 48; University of North Carolina) and rAAV2/AAV-hGFAP-hM3DGq-mCherry (v97; University of Zurich) were applied into each hemisphere (coordinates: bregma, anterior-posterior: $-1.2 \mathrm{~mm}$, dorsal-ventral: $-5.8 \mathrm{~mm}$, lateral: $\pm 0.3 \mathrm{~mm}$ ) by using an air pressure system (injection time: 5 minutes). After surgery, mice (Gfap-gfp KORD ${ }^{A g R P}$ and $N p y$ - $g f p h M 3 D_{G q}{ }_{G F A P}$ ) were allowed to recover for 3-4 weeks before electrophysiological recording. After the electrophysiological experiments were finished, brain sections were fixed with $4 \% \mathrm{PFA}$, and $50-\mu \mathrm{m}$-thick vibratome sections of the ARC were prepared. Accurate virus injection into the ARC was verified by analyzing local GFP fluorescence. Mice with "missed" or "partial" hits were excluded. Specific (virus) expression in ARC was signified by double fluorescence labeling for GFP and GFAP.

Ghrelin administration. After removal of food at 8:30 am, 20 nmol/200 $\mu$ L Human Recombinant Ghrelin (HOR-294, ProSpec Bio) or $200 \mu \mathrm{L}$ saline was injected i.p., and 90 minutes later mice were sacrificed according to the IACUC protocols. For Western blotting purposes, MBHs were extracted and processed (see Western blot analysis). For electron microscopy analyses, mice were anesthetized and perfused (see Electron microscopy and mitochondrial analysis).

CNO administration. $h M 3 D_{G q}{ }_{G g R P}$ mice were treated with $\mathrm{CNO}(0.3$ $\mu \mathrm{g} / \mathrm{kG})(4936$, Tocris) twice daily for 5 days. On the last day of treatment, food was removed from the cage, and the mice were sacrificed 90 minutes after CNO administration according to the IACUC protocols. As a control, we used both $h M 3 D_{G q}{ }^{A g R P}$ mice treated with saline or WT mice treated with CNO. For Western blotting purposes, MBHs were extracted and processed (see Western blot analysis). For electron microscopy analyses, mice were anesthetized and perfused (see Electron microscopy and mitochondrial analysis). $h M 4 D_{G i}{ }^{A g R P}$ mice were treated with CNO $(0.3 \mu \mathrm{g} / \mathrm{kG})$ (4936, Tocris) or saline 15 minutes prior the administration of ghrelin or saline. Feeding was assessed 2 hours after i.p. injection of ghrelin. For Western blotting purposes, brains were extracted and processed (see Western blot analysis).

SalB administration. Agrp-cre mice infected with rAAV9/AAV-HAKORD-IRES-mCitrine ( $G f a p$ - $g f p$ KORD ${ }^{A g R P}$ ) were used for electrophysiological purposes. Prior to the experiments, feeding was assessed in all mice. For this, mice were overnight fasted. Mice were injected i.p. with vehicle or $5 \mathrm{mg} / \mathrm{kg}$ SalB (11487, Cayman) between 8 am and 9 am, and 10 minutes later food was given. Food intake was measured 1 hour after the beginning of the refeeding.

PGE2 administration. Cannulated Npy-gfp mice received a single injection of 100 pmol PGE2 in $2 \mu \mathrm{L}$ vehicle. PGE2 (2246, Tocris) was dissolved in DMSO to produce a stock solution of $5 \mathrm{mg} / \mathrm{mL}$ and diluted in saline until final concentration was achieved. Mice were sacrificed 90 minutes after PGE2 administration.

PFO4418948 (i.c.v.) and ghrelin (i.p.) experiment. Previously cannulated mice received a single injection of PF04418948 (100 pm/2 $\mu \mathrm{L})$ or vehicle. Fifteen minutes later, i.p. ghrelin or vehicle was administered. PF0441894 (15016, Cayman) was dissolved in DMSO to produce a stock solution, and then diluted in saline until final concentration was achieved. Food intake was measured 1 hour after ghrelin administration. For sample collection, mice were sacrificed 90 minutes after ghrelin treatment.

Electron microscopy and mitochondrial analysis. Mice (at least 4 per group) were anesthetized and transcardially perfused with freshly prepared $4 \%$ PFA and $0.1 \%$ glutaraldehyde. After postfixation overnight, vibratome sections $(50 \mu \mathrm{m})$ containing the ARC were immunostained with primary antibody anti-GFP (dilution 1:4000, ab13970, Abcam) or anti-GFAP (dilution 1:4500, G3893, Sigma-Aldrich). After overnight incubation at room temperature, sections were washed with $\mathrm{PB}$, incu- 
bated with biotin-conjugated donkey anti-chicken $\operatorname{IgG}$ (dilution 1:250; 703-065-155, Jackson ImmunoResearch Laboratories Inc.) or donkey anti-mouse IgG (dilution 1:250; 715-065-151, Jackson ImmunoResearch Laboratories Inc.) secondary antibody, respectively; and for 2 hours, washed again, put in avidin-biotin complex (ABC; Vector Laboratories), and developed with DAB. Sections were then osmicated (15 minutes in $1 \%$ osmium tetroxide) and dehydrated in increasing ethanol concentrations. During the dehydration, $1 \%$ uranyl acetate was added to the $70 \%$ ethanol to enhance ultrastructural membrane contrast. Flat embedding in Durcupan (Electron Microscopy Sciences) followed dehydration. Ultrathin sections were cut on a Leica Ultramicrotome, collected on Formvar-coated single-slot grids, and analyzed with a Tecnai 12 Biotwin electron microscope (FEI) with an AMT XR-16 camera $(15,16)$.

Quantification of mitochondria . Hypothalamic sections containing GFP- or GFAP-immunoreactive cells with a visible nucleus were analyzed by electron microscopy. Mitochondrial cross-sectional area, perimeter, and aspect ratio were calculated using ImageJ (NIH). Probability plots were utilized to estimate changes in mitochondrial size and shape, and statistical differences were tested using the Kolmogorov-Smirnov test. Mitochondrial density was estimated by dividing the number of mitochondrial profiles by the cytosolic or cellular area. Mitochondrial coverage was estimated by dividing the total area of mitochondria by the cytosolic or cellular area. Differences in mitochondrial density and coverage were tested using $t$ test. For glial coverage and synaptic inputs, an investigator blinded to the protocol scored the number of synapses and the percentage of glia per AgRP cell in high-magnification images $(>4.800 \times$; refs. 15,16$) . P \leq 0.05$ was considered statistically significant.

Immunofluorescence. Postfixed sections were cut into $50-\mu \mathrm{m}$-thick sections. After 15 minutes washing in $\mathrm{PB}$, the sections were incubated in blocking solution (1:20 normal donkey serum in $\mathrm{PB}$ ) containing $0.2 \%$ Triton $\mathrm{X}-100$ for 30 minutes at room temperature. Sections were incubated with anti-GFAP (dilution 1:2000; G3893, Sigma-Aldrich), anti-GFP (dilution 1:2000; ab13970, Abcam), and anti-c-Fos (dilution 1:1000; sc-52-G, Santa Cruz Biotechnology Inc.) overnight at room temperature. The next day, sections were washed 3 times ( 5 minutes) in $\mathrm{PB}$ and incubated with the respective secondary antibodies for 1 hour at room temperature: donkey anti-mouse IgG Fluor 594; A-21203 (dilution 1:500); goat anti-chicken IgG Fluor 488, A-11039 (1:500); and goat anti-rabbit IgG 594, A-11012 (1:500) (Life Technologies). The sections were coverslipped and scoped using a Keyence BZ-X700 fluorescence microscope.

Western blot analysis. Mice ( $n=6-7$ per group) were sacrificed, and the $\mathrm{MBH}$ was extracted. Protein was extracted using RIPA buffer containing Complete Protease Inhibitor Cocktail (Roche). Proteins were transferred onto PVDF membranes using a Trans-Blot Turbo transfer apparatus (Bio-Rad) and incubated with anti-GFAP (dilution 1:2000; G3893, Sigma-Aldrich), anti-pDRP1 (1:1000; 3455, Cell Signaling Technology), and anti-GAPDH (1:10,000; cb1001, Calbiochem). Detection was carried out using ECL (Bio-Rad).

RT-PCR and ribosome profiling. RNA was extracted using a QIAGEN RNeasy Micro Kit (no. 74004). cDNA was synthetized using a QIAGEN Whole Transcriptome Kit (no. 207043). RT-PCR was performed in a Roche 480 LightCycler using Taqman probes (Gabarap; Mm00490680_m1).

Translating ribosome affinity purification (TRAP) was conducted in homogenate samples of $\mathrm{MBH}$ obtained from mice with
loxP-flanked ribosome protein subunit $22(\mathrm{Rpl} 22)$ crossed with the Gfap-cre line (011029 and 012849, respectively, The Jackson Laboratory), which express Rpl22 and HA proteins in ribosomes of astrocytes, thereby allowing for the immunoprecipitation of polysomes directly from GFAP-positive astrocytes (15). After RNA isolation, we obtained approximately 10-25 ng RNA per sample. RT-PCR was performed as described above.

Electrophysiology. Coronal hypothalamic slices containing the ARC were prepared from Gfap-gfp, Gfap-gfp $h M 3 D_{G q}{ }^{A g R P}, G f a p-g f p$ $K O R D^{A g R P}$, or $N p y-g f p h M 3 D_{G q}^{G F A P}$ mice as previously reported (15, 49). Briefly, mice were anesthetized with isoflurane and decapitated, and the brain was rapidly removed and immersed in cold $\left(4^{\circ} \mathrm{C}\right)$ and oxygenated cutting solution containing $(\mathrm{mM})$ : sucrose $220, \mathrm{KCl} 2.5$, $\mathrm{NaH}_{2} \mathrm{PO}_{4} 1.23, \mathrm{NaHCO}_{3} 26, \mathrm{CaCl}_{2} 1, \mathrm{MgCl}_{2} 6$, and glucose 10 (pH 7.3 with $\mathrm{NaOH})$. Coronal hypothalamic slices $(300 \mu \mathrm{m}$ thick) were prepared with a Leica vibratome after the brain was trimmed to a small tissue block containing the hypothalamus. After preparation, slices were maintained at room temperature $\left(23^{\circ} \mathrm{C}-25^{\circ} \mathrm{C}\right)$ in a storage chamber in artificial cerebrospinal fluid (ACSF) (bubbled with $5 \% \mathrm{CO}_{2}$ and $95 \% \mathrm{O}_{2}$ ) containing (in $\mathrm{mM}$ ): $\mathrm{NaCl} 124, \mathrm{KCl} 3, \mathrm{CaCl}_{2} 2, \mathrm{MgCl}_{2} 2$, $\mathrm{NaH}_{2} \mathrm{PO}_{4} 1.23, \mathrm{NaHCO}_{3} 26$, glucose 10 (pH 7.4 with $\mathrm{NaOH}$ ) for recovery and storage. After recovery at room temperature for at least 1 hour, slices were transferred to a recording chamber constantly perfused at a rate of $2 \mathrm{~mL} / \mathrm{min}$ with ACSF containing $2.5 \mathrm{mM}$ glucose at a temperature of $33^{\circ} \mathrm{C}$ for electrophysiological experiments. Whole-cell patch clamp recording was performed in GFAP-GFP-positive cells, and spontaneous membrane (MP) was recorded under current clamp. The micropipettes (4-6 M $\Omega$ ) were made of borosilicate glass (World Precision Instruments) with a micropipette puller (Sutter P-97) and backfilled with a pipette solution containing (in $\mathrm{mM}$ ): K-gluconate 108, $\mathrm{KCl} 27, \mathrm{MgCl}_{2}$ 2, HEPES 10, EGTA 1.1, Mg-ATP 2.5, $\mathrm{Na}_{2}$-GTP 0.3, and $\mathrm{Na}_{2}$-phosphocreatine 10, $\mathrm{pH} 7.3$ with $\mathrm{KOH}$. Both input resistance and series resistance were monitored throughout the experiments, and the former was partially compensated. Only recordings with stable series resistance and input resistance were accepted. All data were sampled at $3 \mathrm{kHz}$, filtered at $3 \mathrm{kHz}$, and analyzed with an Apple Macintosh computer using AxoGraph X. $t$ test or 1-way ANOVA was used to examine the statistical significance of the difference in MP in the recorded glial cells or NPY neurons.

Statistics. All data are expressed as mean \pm SEM. The differences in means between 2 groups were analyzed by Student's $t$ tests. For experiments with experimental groups and 2 conditions, 2-way-ANOVA with post-hoc Tukey's test was performed. For analyses of populations (cumulative distributions), Kolmogorov-Smirnov test was used. For electrophysiological experiments comparing the same cell within different conditions, paired 2-tailed $t$ test or repeated-measures 1-way ANOVA was performed. $P \leq 0.05$ was considered statistically significant. Shapiro-Wilk normality test was used to assess the Gaussian distribution of each data set. All data sets show normal distribution.

Study approval. All experimental procedures were performed in accordance with Yale Animal Resources Center (YARC) policies and protocols approved by the Yale IACUC.

\section{Author contributions}

LV, XBG, and ZWL performed the experiments. BS performed stereotaxic surgeries. JES and JGK acquired data. LV and TLH designed the study. LV and TLH wrote and edited the manuscript. 
LV and TLH are the guarantors of this work and, as such, had full access to all the data in the study, and take responsibility for the integrity of the data and the accuracy of the data analysis.

\section{Acknowledgments}

This work was supported by NIH grants AG052005, AG052986, AG051459, and DK111178 (to TLH), DA046160 (TLH and XBG), and DK120891 (XBG and TLH); and grant NKFI-126998 from the Hungarian National Research, Development, and Innovation Office (TLH).

Address correspondence to: Luis Varela or Tamas Horvath, Department of Comparative Medicine, Yale School of Medicine, 310 Cedar St. BML 330, New Haven, Connecticut 06520, USA. Phone: 203.785.2525; Email: luis.varela@yale.edu (LV); tamas. horvath@yale.edu (TH).
1. Pinto S, et al. Rapid rewiring of arcuate nucleus feeding circuits by leptin. Science. 2004;304(5667):110-115.

2. Horvath TL. The hardship of obesity: a soft-wired hypothalamus. Nat Neurosci. 2005;8(5):561-565.

3. Horvath TL, Diano S. The floating blueprint of hypothalamic feeding circuits. Nat Rev Neurosci. 2004;5(8):662-667.

4. Suyama S, et al. Plasticity of calcium-permeable AMPA glutamate receptors in Pro-opiomelanocortin neurons. Elife. 2017;6:e25755.

5. Abizaid A, et al. Ghrelin modulates the activity and synaptic input organization of midbrain dopamine neurons while promoting appetite. J Clin Invest. 2006;116(12):3229-3239.

6. Diano S, et al. Ghrelin controls hippocampal spine synapse density and memory performance. Nat Neurosci. 2006;9(3):381-388.

7. Andrews ZB, et al. UCP2 mediates ghrelin's action on NPY/AgRP neurons by lowering free radicals. Nature. 2008;454(7206):846-851.

8. Horvath TL, et al. Synaptic input organization of the melanocortin system predicts diet-induced hypothalamic reactive gliosis and obesity. Proc Natl Acad Sci U S A. 2010;107(33):14875-14880.

9. Sternson SM, et al. Topographic mapping of $\mathrm{VMH} \rightarrow$ arcuate nucleus microcircuits and their reorganization by fasting. Nat Neurosci. 2005;8(10):1356-1363.

10. Kong D, et al. A postsynaptic AMPK--> p21activated kinase pathway drives fasting-induced synaptic plasticity in AgRP neurons. Neuron. 2016;91(1):25-33.

11. Yang $\mathrm{Y}$, et al. Hunger states switch a flip-flop memory circuit via a synaptic AMPK-dependent positive feedback loop. Cell. 2011;146(6):992-1003.

12. Abizaid A, Horvath TL. Brain circuits regulating energy homeostasis. Regul Pept. 2008;149(1-3):3-10.

13. Dietrich MO, et al. Agrp neurons mediate Sirt1's action on the melanocortin system and energy balance: roles for Sirt1 in neuronal firing and synaptic plasticity. J Neurosci. 2010;30(35):11815-11825.

14. Liu T, et al. Fasting activation of AgRP neurons requires NMDA receptors and involves spinogenesis and increased excitatory tone. Neuron. 2012;73(3):511-522.

15. Kim JG, et al. Leptin signaling in astrocytes regulates hypothalamic neuronal circuits and feeding. Nat Neurosci. 2014;17(7):908-910.

16. Garcia-Caceres C, et al. Astrocytic insulin signaling couples brain glucose uptake with nutrient availability. Cell. 2016;166(4):867-880.
17. Tschop M, et al. Ghrelin induces adiposity in rodents. Nature. 2000;407(6806):908-913.

18. Cummings $\mathrm{DE}$, et al. A preprandial rise in plasma ghrelin levels suggests a role in meal initiation in humans. Diabetes. 2001;50(8):1714-1719.

19. Liu T, et al. Action of neurotransmitter: a key to unlock the AgRP neuron feeding circuit. Front Neurosci. 2013;6:200.

20. Clasadonte J, et al. Prostaglandin E2 release from astrocytes triggers gonadotropinreleasing hormone $(\mathrm{GnRH})$ neuron firing via EP2 receptor activation. Proc Natl Acad Sci U S A. 2011;108(38):16104-16109.

21. Rage F, et al. Estradiol enhances prostaglandin E2 receptor gene expression in luteinizing hormone-releasing hormone (LHRH) neurons and facilitates the LHRH response to PGE2 by activating a glia-to-neuron signaling pathway. J Neurosci. 1997;17(23):9145-9156.

22. Chen N, et al. Direct modulation of GFAPexpressing glia in the arcuate nucleus bi-directionally regulates feeding. Elife. 2016;5:e18716.

23. Araque A. Astrocytes process synaptic information. Neuron Glia Biol. 2008;4(1):3-10.

24. Perea G, et al. Tripartite synapses: astrocytes process and control synaptic information. Trends Neurosci. 2009;32(8):421-431.

25. Araque A, Navarrete M. Glial cells in neuronal network function. Philos Trans R Soc Lond B Biol Sci. 2010;365(1551):2375-2381.

26. Perez-Alvarez A, Araque A. Astrocyte-neuron interaction at tripartite synapses. Curr Drug Targets. 2013;14(11):1220-1224.

27. Ben Haim L, Rowitch DH. Functional diversity of astrocytes in neural circuit regulation. Nat Rev Neurosci. 2017;18(1):31-41.

28. Allen NJ, Eroglu C. Cell biology of astrocyte-synapse interactions. Neuron. 2017;96(3):697-708.

29. Ioannou MS, et al. Neuron-astrocyte metabolic coupling protects against activity-induced fatty acid toxicity. Cell. 2019;177(6):1522-1535.

30. Fuente-Martin E, et al. Leptin regulates glutamate and glucose transporters in hypothalamic astrocytes. J Clin Invest. 2012;122(11):3900-3913.

31. Navarrete M, Araque A. Endocannabinoids mediate neuron-astrocyte communication. Neuron. 2008;57(6):883-893.

32. Fellin T, Carmignoto G. Neurone-to-astrocyte signalling in the brain represents a distinct multifunctional unit. J Physiol. 2004;559(pt 1):3-15.

33. Araque A, et al. Synaptically released acetylcholine evokes $\mathrm{Ca} 2+$ elevations in astrocytes in hippocam- pal slices. J Neurosci. 2002;22(7):2443-2450.

34. Losi G, et al. GABAergic interneuron to astrocyte signalling: a neglected form of cell communication in the brain. Philos Trans R Soc Lond B Biol Sci. 2014;369(1654):20130609.

35. Velez-Fort M, et al. Central role of GABA in neuron-glia interactions. Neuroscientist. 2012;18(3):237-250.

36. Kang J, et al. Astrocyte-mediated potentiation of inhibitory synaptic transmission. Nat Neurosci. 1998;1(8):683-692.

37. Forsberg D, et al. Astrocytes release prostaglandin E2 to modify respiratory network activity. Elife. 2017;6:e29566.

38. Krashes MJ, et al. Rapid, reversible activation of AgRP neurons drives feeding behavior in mice. J Clin Invest. 2011;121(4):1424-1428.

39. Dietrich MO, et al. Hypothalamic Agrp neurons drive stereotypic behaviors beyond feeding. Cell. 2015;160(6):1222-1232.

40. Li C, et al. AGRP neurons modulate fasting-induced anxiolytic effects. Transl Psychiatry. 2019;9(1):111.

41. Padilla SL, et al. Agouti-related peptide neural circuits mediate adaptive behaviors in the starved state. Nat Neurosci. 2016;19(5):734-741.

42. Peyrache A, et al. Inhibition recruitment in prefrontal cortex during sleep spindles and gating of hippocampal inputs. Proc Natl Acad Sci U S A. 2011;108(41):17207-17212.

43. Chamberland S, Topolnik L. Inhibitory control of hippocampal inhibitory neurons. Front Neurosci. 2012;6:165.

44. Melzer S, et al. Long-range-projecting GABAergic neurons modulate inhibition in hippocampus and entorhinal cortex. Science. 2012;335(6075):1506-1510.

45. Freund TF, Antal M. GABA-containing neurons in the septum control inhibitory interneurons in the hippocampus. Nature. 1988;336(6195):170-173.

46. Horvath TL, Gao XB. Input organization and plasticity of hypocretin neurons: possible clues to obesity's association with insomnia. Cell Metab. 2005;1(4):279-286.

47. Steculorum SM, et al. AgRP neurons control systemic insulin sensitivity via myostatin expression in brown adipose tissue. Cell. 2016;165(1):125-138.

48. Vardy E, et al. A new DREADD facilitates the multiplexed chemogenetic interrogation of behavior. Neuron. 2015;86(4):936-946.

49. Varela L, et al. Endothelial HIF-1 $\alpha$ enables hypothalamic glucose uptake to drive POMC neurons. Diabetes. 2017;66(6):1511-1520. 